\title{
La influencia de la musicoterapia en la mejora de la precisión y de la velocidad lectora en niños y niñas de edades comprendidas entre 6 y 9 años escolarizados con dificultades de aprendizaje
}

Sophie Moutte ${ }^{1}$

\author{
Recibido: 10/10/2019 Aceptado: 12/12/2019
}

\begin{abstract}
RESUMEN
En la actualidad existen numerosos estudios cuyos resultados han demostrado la eficacia de las intervenciones musicales para fomentar las habilidades del lenguaje oral y de la lectoescritura. Música y lenguaje requieren habilidades similares, tales como la discriminación auditiva o el sentido del ritmo. En esta observación participante el objetivo fue el de analizar el impacto de las sesiones de musicoterapia sobre la mejora de la precisión y de la velocidad lectora en niños y niñas escolarizados con dificultades de aprendizaje de edades comprendidas entre los 6 y los 9 años. Los participantes del estudio eran dos niñas de 8 y 9 años de un grupo de tres alumnas y dos niños y una niña de 6 años de un grupo de cinco usuarios. Cada grupo recibió una sesión semanal de 45 minutos durante tres meses. Para poder medir la evolución de los participantes se evaluó el nivel de precisión y la velocidad lectora de cada uno de ellos mediante una prueba realizada antes y después del periodo de intervención con musicoterapia. Los resultados obtenidos mostraron una mejora considerable de los participantes en su precisión y en su velocidad lectora. Pero hay que tener en cuenta que sus progresos son también debidos a todo el trabajo realizado por el alumnado durante ese periodo, fuera de las sesiones de musicoterapia. Se puede concluir, por lo tanto, que la musicoterapia puede contribuir a la mejora de la precisión y de la velocidad lectora dentro de un contexto escolar.
\end{abstract}

Palabras clave: musicoterapia, habilidades lectoras, dificultades de aprendizaje, conciencia fonológica

\section{The influence of music therapy sessions on the improvement of accuracy and reading speed in school children with learning difficulties aged 6-9 \\ ABSTRACT}

Today there are numerous studies whose results have demonstrated the effectiveness of musical interventions to promote oral language and literacy skills. Music and language both require similar skills, such as auditory discrimination or sense of rhythm. In this participant observation the objective was to see the impact of music therapy sessions on the improvement of accuracy and reading speed in school children with learning difficulties aged 6-9. The study participants were one 8-year-old girl and one 9-year-old girl from a group of three students and two boys and one girl of 6 years of age from a group of five users. Each group received a weekly 45-minute session for three months. In order to measure the participants' progress, the level of accuracy and reading speed of each participant, a test was carried out before and after the intervention period with music therapy. The results obtained showed a considerable improvement in the participants' accuracy and reading speed. But it is important to keep in mind that their progress is also due to all the work done by the students during that period, outside the music therapy sessions. It can be concluded, therefore, that music therapy can contribute to the improvement of accuracy and reading speed within a school context.

Keywords: music therapy, learning disabilities, reading skills, reading disabilities, phonological awareness

\footnotetext{
${ }^{1}$ Profesora de Flauta y Música y Movimiento de la Escuela municipal de música Isaac Albéniz de Madrid
} 
Sumario. 1. Introducción.2. Método.3. Resultados 4. Discusión 5. Conclusiones 6. Referencias

Cómo citar. Moutte, S. (2020). La influencia de la musicoterapia en la mejora de la precisión y de la velocidad lectora en niños y niñas de edades comprendidas entre 6 y 9 años escolarizados con dificulta des de aprendizaje. Revista de Investigación en Musicoterapia, 3,21-33 doi.org/10.15366/rim2019.3.002

\section{Introducción}

Existen varios factores por los que el aprendizaje de la lectura puede suponer un reto especialmente grande para ciertos niños y niñas. Entre estos factores destacan las dificultades de aprendizaje, en adelante DDAA, que, según Martínez, Henao y Gómez (2009), son las principales causas del fracaso escolar, indicando que 8 de cada 10 estudiantes con DDAA obtienen resultados académicos poco positivos.

El DSM V-TM (2013) clasifica las dificultades de aprendizaje como un trastorno específico del aprendizaje, en el que se incluyen las dificultades en la lectura, en la expresión escrita y en las matemáticas. La definición de las dificultades de aprendizaje de Santiuste y GonzálezPérez (2005), destacada por Fuiza y Fernández (2013) es la siguiente:

Las DDAA se definen como aquellas dificultades de aprendizaje que están constituidas por un conjunto heterogéneo de problemas cuyo origen es, probablemente, una disfunción del sistema nervioso central. Se manifiesta primariamente con problemas en el ámbito lingüístico y con defectos de procesamiento en los principales factores cognitivos (atención, percepción, memoria), derivadamente, en el ámbito de las disciplinas instrumentales básicas (lectura, escritura, matemáticas) y, secundariamente, en las diversas áreas curriculares (ciencias experimentales, ciencias sociales, segundo idioma). Cursan, además, con problemas de personalidad, y puede ocurrir a lo largo del ciclo vital del sujeto. (p. 25)

El alumnado con DDAA puede presentar alteraciones en su percepción auditiva, las cuales conllevan dificultades de dicción, de discriminación de sonidos, de identificación de rimas, así como para marcar un pulso. Todas estas dificultades influyen directamente sobre la conciencia fonológica de dichos niños y niñas. La mejora de la conciencia fonológica conduce a una lectura más fluida, lo cual permitiría, a su vez, una mejor comprensión lectora (Fuiza y Fernández, 2013).

Durante la lectura, se puede recurrir a dos rutas distintas: la ruta visual (directa), que consiste en descodificar una palabra según su representación gráfica, y la ruta fonológica (indirecta), que atribuye un sonido a cada letra (Sánchez e Hidalgo, 1990). Schwartz (1984) (como se citó en Sánchez e Hidalgo, 1990) considera que es preferible que los niños y las niñas hagan 
uso de ambas rutas para realizar un aprendizaje adecuado de la lectura. La ruta fonológica implica la adquisición de la conciencia fonológica, la cual corresponde a unas habilidades metalingüísticas y se organiza en distintos niveles. A lo largo de los años, ha habido varios intentos para clasificar estos niveles. Adams (1990), plantea las tareas de conciencia fonológica por orden de dificultad, estableciendo cinco tipos de tareas:

1. Reconocer rimas sencillas.

2. Identificar rimas y aliteraciones en un texto.

3. Juntar o separar un elemento de una sílaba.

4. Dividir una palabra en sus distintos fonemas.

5. Crear una nueva palabra, omitiendo, añadiendo o invirtiendo fonemas de la palabra original.

Otra forma de clasificar dichas tareas es la propuesta por Treiman (1991), quien divide la conciencia fonológica en tres niveles, también por orden de dificultad. En primer lugar, hace alusión a la conciencia silábica, a la cual sigue la conciencia intrasilábica y finaliza por la conciencia fonémica.

A pesar de sus diferencias, ambos modos de clasificar los grados de conciencia fonológica coinciden en que la segmentación silábica es una de las primeras etapas en el desarrollo de la misma, y es un tipo de actividad que se emplea con frecuencia para trabajar este aspecto. Esta actividad consiste en dar palmadas al mismo tiempo que se pronuncia cada sílaba de una palabra con el objetivo de identificar cada una de ellas, para así poder distinguir las unas de las otras. Aquí es donde entra en juego la dimensión rítmica. Con respecto al ritmo, existen numerosos estudios (Corriveau y Goswami, 2009; Thomson y Goswami, 2008; Tierney y Kraus, 2013) que ponen de manifiesto que el hecho de marcar un pulso isocrónico requiere de un esfuerzo especial para las personas con dificultades en el lenguaje y en la lectura. En este sentido, Goswami (2013) sugiere que los movimientos rítmicos sincronizados con uso del lenguaje y con la música podrían ser beneficiosos para las personas que lo ejerciten. Asimismo, el ritmo determina la estructura de la música y a su vez la del lenguaje; siendo un elemento clave para la inteligibilidad del discurso y es el que nos permite distinguir un fonema dentro de una sílaba, una sílaba dentro de una palabra, una palabra dentro de una frase y una frase dentro de un texto (Bonacina, Krizman, White-Schwoch, Nicol y Kraus, 2019).

La conciencia fonológica es una habilidad metalingüística indispensable para el aprendizaje de la lectura, con lo cual, si la musicoterapia influye en su desarrollo, influye 
también en las habilidades lectoras y a su vez en el incremento de la autoestima, a menudo baja en niños y niñas con DDAA.

\section{Líneas de investigación}

Sallat y Jentschke (2015), investigaron las dificultades que tienen los niños y niñas con trastorno específico del lenguaje a la hora de discriminar el tono y el ritmo de una frase musical; dichas dificultades podrían explicar los problemas que encuentran los mismos en su proceso de adquisición del lenguaje.

Con respecto a la relación que puede existir entre la música y la adquisición del lenguaje, Lorenzo, Herrera, Hernández-Candelas y Badea (2014) compararon dos grupos de niños y niñas de 3 y 4 años que participaban en un programa innovador en la ciudad de Puerto Rico. El grupo experimental recibió un mínimo de 3 clases de música de 20 minutos a la semana durante dos años, mientras que el grupo control no recibió ninguna clase de música. Para poder evaluar el impacto de la música en la adquisición del lenguaje, realizaron un pre y post test en ambos gupos, cuyos resultados demostraron que la música puede tener un rol significativo en el desarrollo del lenguaje de los niños y de las niñas.

Desde otra perspectiva, Talamini, Grassi, Toffalini, Santoni y Carretti (2018) se interesaron en el vínculo que podría existir entre la práctica musical, las aptitudes musicales y el aprendizaje de una lengua extranjera (inglés) en alumnos y alumnas italianos de edades comprendidas entre 11 y 15 años. De los ochenta participantes, cuarenta y uno de ellos fueron considerados músicos debido a la formación extraescolar que tenían, mientras que el resto fue considerado no músico. El grupo que practicaba la música presentó resultados significativamente más altos que el otro en las pruebas de dictado en inglés. La conclusión de este estudio fue que la práctica de la música podía influir en el aprendizaje de un idioma extranjero porque ambos requieren de una capacidad de discriminación auditiva.

\section{Antecedentes}

Siguiendo esta línea continuista, Anvari, Trainor, Woodside y Levy (2002) estudiaron la relación entre la iniciación musical, el proceso fonológico y el aprendizaje de la lectura en niños y niñas de 4 y 5 años de edad. Los resultados obtenidos les permitieron afirmar que existía una relación directa entre estos tres elementos y que, por lo tanto, la música podía influir en el desarrollo de la capacidad lectora. La conciencia fonológica requiere la habilidad 
de segmentar el discurso en sus componentes sonoros y de categorizar estos mismos según el tono, el ritmo, el contexto y el locutor. De la misma manera, en la música el oyente necesita poder discriminar un sonido de otro según el tono, el tempo, el intérprete y el contexto.

En un ámbito similar, Moritz, Yampolsky, Papadelis, Thomson y Wolf (2013) se preguntaron si habría un vínculo entre la conciencia fonológica y las habilidades rítmicas de niños de 5 años en escuelas infantiles. También quisieron observar si aquellos y aquellas que hubiesen asistido a clases musicales intensivas presentarían una conciencia fonolófica más desarrollada que los que hubiesen recibido menos estímulos musicales. Tras la investigación, pudieron afirmar que las capacidades rítmicas de los niños y de las niñas estaban directamente ligadas al nivel de conciencia fonológica y que los que habían tenido un contacto más cercano con la música habían presentado una mayor mejoría en su conciencia fonológica que los demás.

\section{Principales proyectos de referencia}

En los últimos años, varios estudios se han enfocado en el rol de la música a la hora de adquirir las habilidades lectoras. Pero hoy en día siguen siendo poco numerosas las investigaciones que se han interesado en el efecto de la musicoterapia (de la terapia en sí y no solo de la música como simple herramienta) con respecto al aprendizaje de la lectura.

Register (2001), comparó los efectos de unas sesiones de musicoterapia diseñadas específicamente para desarrollar la predisposición a la lectura y la escritura en niños y niñas de entre 4 y 5 años con unas sesiones elaboradas para fomentar las habilidades cognitivas, la socialización y el movimiento. El grupo experimental recibió las sesiones directamente relacionadas con el aprendizaje o el fortalecimiento de la lectoescritura, mientras que el grupo control recibió sesiones musicales con un carácter más general, excluyendo a propósito todo tipo de actividades vinculadas con la lectoescritura. Los niños de cada grupo asistieron durante 30 semanas a dos sesiones semanales de 30 minutos cada una. Se emplearon tres tipos de test distintos para medir la evolución en el lenguaje oral y en el escrito antes y después del proceso. Los resultados revelaron una mayor mejoría en el nivel de lectoescritura del grupo experimental, en las tres áreas puestas a prueba, comparado con el grupo control. Estos datos reflejan no solo la influencia positiva que puede tener la música en el proceso de aprendizaje de la lectoescritura en la escuela infantil, sino que demuestran también que las intervenciones musicales diseñadas con un objetivo académico específico tienen mayor efectividad en el desarrollo de dicha área. 
Desde otro vértice, Cross (2014) analizó el uso de la musicoterapia para fomentar el desarrollo de las habilidades lectoras en niños y niñas. Para ello, entrevistó a 11 musicoterapeutas a quienes preguntó qué elementos de la música usaban para trabajar este tema. La mayoría mencionó el ritmo como el elemento clave para dar estructura y ayudar a los usuarios a la hora de realizar una actividad relacionada con la lectura. Entre los varios usos que se pueden hacer del ritmo, mencionaron el tocar una melodía enfatizando el pulso, segmentar las palabras en sílabas y sincronizarlas con los golpes en un instrumento, es decir, jugar con los acentos rítmicos de una frase o de una palabra. Además del ritmo, varios musicoterapeutas nombraron la importancia del apoyo visual para acompañar actividades ligadas a la lectura. Los elementos visuales permiten una mayor concentración por parte de los usuarios y les ayuda a asimilar mejor el aprendizaje realizado. Sin embargo, el autor llegó a la conclusión de que ninguno de los musicoterapeutas había elaborado una forma determinada de intervenir, sino que, debido a las circunstancias de la institución en la que trabajaba cada uno de ellos y al nivel de los usuarios, cada terapeuta necesitaba diseñar unas actividades específicas al caso al que se enfrentaba, y por lo tanto, no se podía hablar de un modelo de intervención general que pudiese funcionar para todos.

\section{Justificación}

La habilidad lectora, a partir de cierta edad, es indispensable para poder desarrollarse de manera adecuada en el marco escolar. Más allá de este ámbito, saber leer permite ampliar el conocimiento, la imaginación o la creatividad. La lectura está directamente vinculada a la escritura. De este modo, los sujetos con problemas de lectura tienden a tener problemas de escritura, y viceversa. Estos y otros factores son los que han generado el interés en saber cómo la musicoterapia puede contribuir en la adquisición y fortalecimiento de estas capacidades.

\section{Problema al que da respuesta}

Desde el prisma del aprendizaje de la lectura, este aprendizaje depende, en buen grado, de la conciencia fonológica, la cual está directamente vinculada a la percepción auditiva. Por lo tanto, los problemas que se presentan para la realización de este trabajo, y a los que se pretende dar respuesta, están relacionados con determinados elementos que conforman la conciencia fonológica y que se pueden trabajar a través de la musicoterapia, así como al estudio en el que esta disciplina puede influir en la mejora de la velocidad lectora. 


\section{Hipótesis}

La musicoterapia contribuye en la mejora de la precisión y de la velocidad lectora en niños y niñas escolarizados con dificultades de aprendizaje de edades comprendidas entre 6 y 9 años.

\section{Objetivo general}

Realizar un Trabajo fin de Máster, según los requisitos exigidos desde el Máster de Musicoterapia de la Facultad de Medicina de la Universidad Autónoma de Madrid para evaluar los resultados obtenidos a través de sesiones de musicoterapia orientadas en la mejora de la precisión y de la velocidad lectora en niños y niñas escolarizados con dificultades de aprendizaje de edades comprendidas entre los 6 y los 9 años.

\section{Objetivos específicos}

1. Promover actividades instrumentales motivadoras que permitan la mejora en la velocidad lectora.

2. Crear actividades vocales que permitan desarrollar la conciencia fonológica.

3. Generar actividades motivadoras que contribuyan en la mejora de la autoestima del alumnado participante en la muestra.

4. Evaluar el impacto de las sesiones de musicoterapia en la velocidad lectora y en el desarrollo de la conciencia fonológica.

\section{Método}

Este trabajo se basa en una observación participante llevado a cabo en un centro educativo del Sureste de la Comunidad de Madrid, durante dos horas semanales de musicoterapia, repartidas en dos sesiones grupales de 45 minutos y una sesión individual de 30 minutos en un marco de prácticas durante un periodo de tres meses. Es un colegio concertado bilingüe de titularidad religiosa que acoge a alumnos desde las etapas de Educación Infantil hasta Bachillerato. El colegio dispone de dos orientadoras, así como de una maestra especialista en pedagogía terapéutica y audición y lenguaje, quien tiene un conocimiento más detallado sobre los distintos diagnósticos de los niños y niñas que presentan ciertas dificultades en el ámbito escolar. 


\section{Participantes}

El estudio se centró en el alumnado de las sesiones grupales que tenían lugar los viernes a mediodía. El primer grupo estaba formado por tres niñas con edades comprendidas entre los 8 y los 9 años, de las cuales solo dos participaron en la investigación. El segundo grupo contaba con cinco niños y niñas de 6 años, de los cuales participaron tres. Algunos alumnos no pudieron participar en las evaluaciones lectoras con lo cual no fueron incluidos en el estudio.

Gracias a los datos obtenidos de la maestra especialista de pedagogía terapéutica, se constató que los participantes de ambos grupos tenían DDAA y todos presentaban problemas en la lectura.

\section{Sesiones de musicoterapia}

Las sesiones combinaron un enfoque humanista inspirado en el modelo NordoffRobbins con respecto al tipo de improvisaciones llevadas a cabo y el modelo cognitivo conductual con el uso de varias actividades estructuradas, enfocadas en la conciencia fonológica.

Las sesiones seguían la siguiente estructura:

- Canción de Bienvenida

- Actividades estructuradas diseñadas para fomentar la conciencia fonológica: canciones centradas en los fonemas, canciones centradas en las sílabas usando la percusión corporal

- Improvisación

- Canción de Despedida

\section{Recopilación de datos}

Para observar la posible influencia de la musicoterapia sobre la mejoría de la precisión y de la velocidad lectora de los niños y las niñas participantes, se realizó una evaluación de lectura antes y después del periodo de intervención con la musicoterapia. La evaluación fue diseñada y dirigida por la musicoterapeuta, quien se apoyó en la prueba diseñada por la Junta de Andalucía y la Consejería de Educación (2010). En esta evaluación se midió la velocidad lectora, cronometrando el número de palabras que era capaz de leer el alumnado en un 
minuto y, mientras leía el texto, se apuntaron los errores, así como el tipo de errores que cometía. Los errores fueron clasificados en dos categorías: errores puntuables y errores no puntuables (ver Anexo). Con el total de errores puntuables y el número de palabras leídas en un minuto se pudo determinar el porcentaje de precisión lectora de cada participante. Se registraron dichos datos en una hoja de cálculo para poder realizar unos gráficos comparativos del antes y del después (ver Anexo). Según el modo de lectura de cada niño o niña, se apuntó si realizaba una lectura expresiva, corriente, vacilante o silábica.

\section{Análisis de Datos}

La mayoría de los participantes cometieron el mismo tipo de errores en ambas evaluaciones. Por ejemplo, dentro de los errores puntuables, uno de los niños de 6 años que había cometido 5 sustituciones y 3 invenciones en la primera evaluación, cometió solo 2 sustituciones y 1 invención en la segunda evaluación. Todos y todas realizaron menos errores no puntuables en la segunda evaluación. Pero en cuanto a los errores puntuables, dos niñas de 8 y 9 años cometieron más errores puntuables en la segunda.

De acuerdo con la reducción general de los errores de lectura (puntuables y no puntuables) se aprecia un avance notable del alumnado tanto en la precisión como en la velocidad lectora entre la primera y la segunda evaluaciones. En cuanto al modo de lectura, no hubo ningún cambio entre las dos evaluaciones para ningún participante.

\section{Resultados}

Los errores no puntuables (salto de líneas, repetición, movimientos de cabeza...) suelen interrumpir el flujo de la lectura. Por lo tanto, su disminución en la segunda evaluación explica el incremento de la precisión y, a su vez, de la velocidad lectora. El alumnado ha sido capaz de leer más palabras en menos tiempo, cometiendo menos errores puntuables, pero del mismo tipo que en su primera evaluación.

La ausencia de evolución en el modo de lectura puede ser debido al hecho de que el modo de lectura es un parámetro mucho más general. Su desarrollo depende del progreso del conjunto de las habilidades lectoras y, por lo tanto, un periodo de tres meses puede que no sea suficiente para contemplar una mejora en este. 


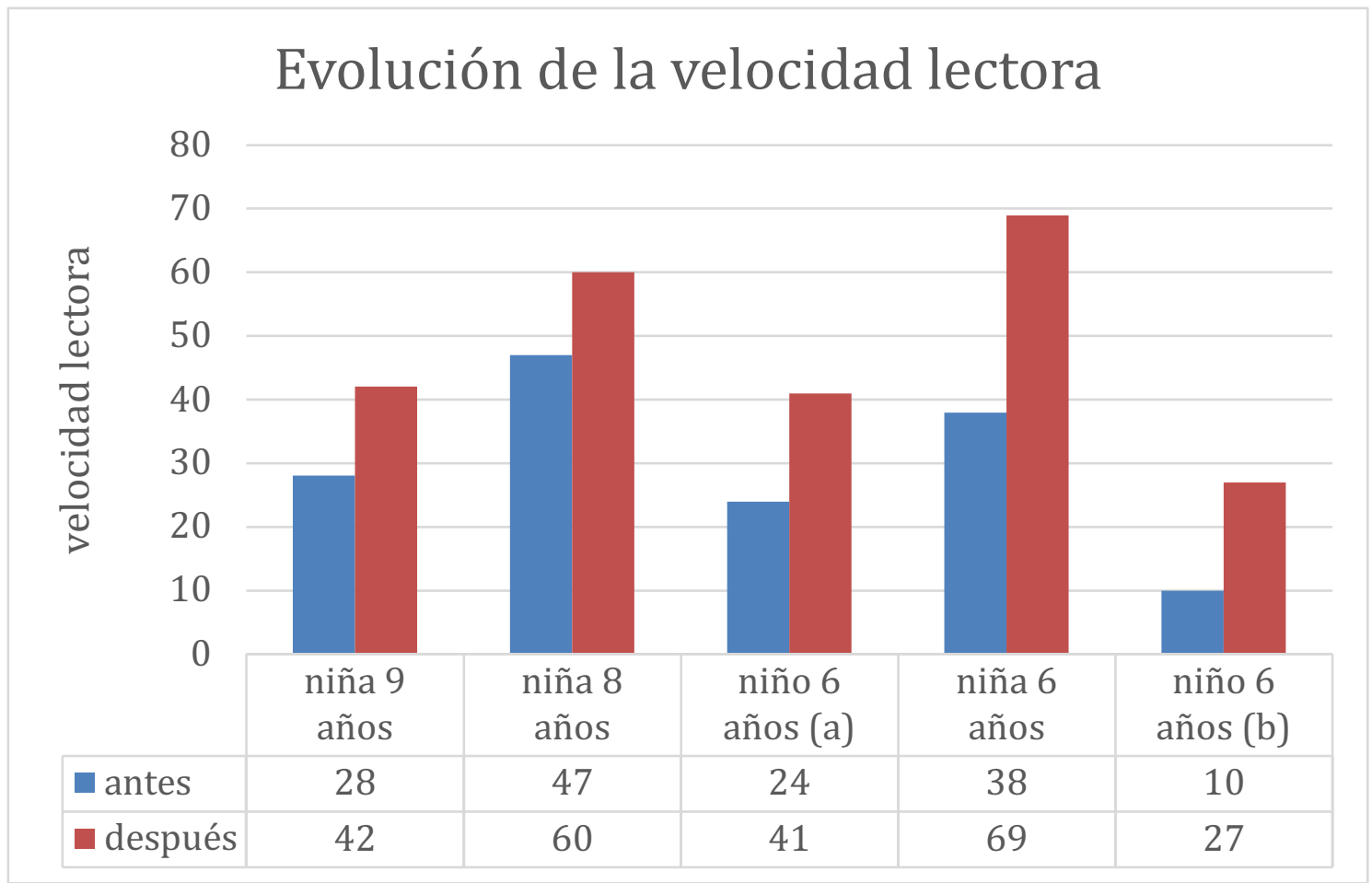

Figura I. Evolución de la velocidad lectora

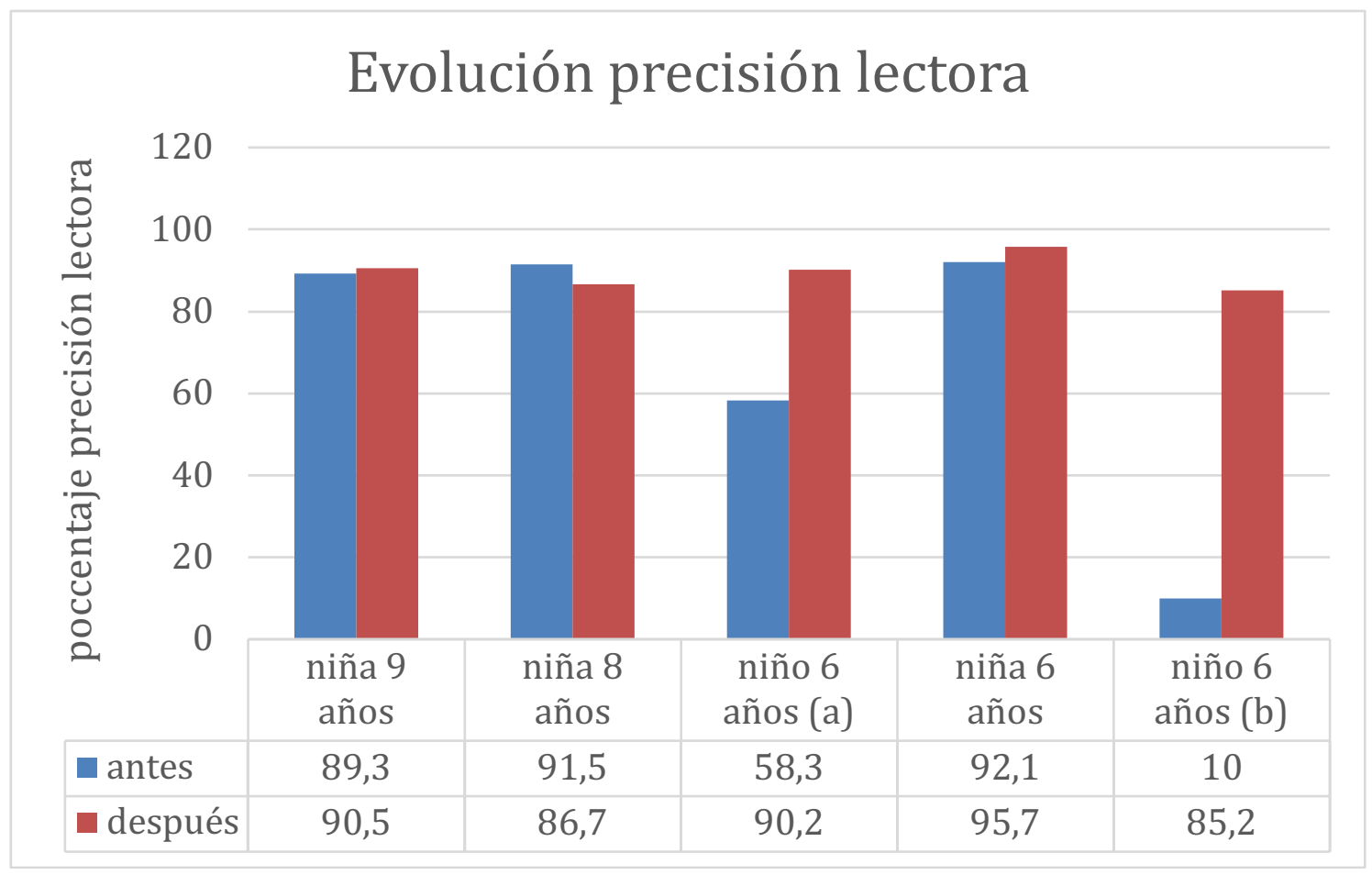

Figura II. Evolución de la precisión lectora 


\section{Discusión}

De acuerdo con los estudios anteriores, los resultados de esta observación participante confirman la posible influencia de la música sobre las habilidades lectoras. En este caso no se hizo simplemente uso de la música en general, sino que se diseñaron con ella unas actividades específicas con unos objetivos específicos. Los resultados obtenidos con este trabajo corroboran los del estudio de Register (2001), quien demostró que las intervenciones musicales planteadas con un objetivo académico propio tienen mayor eficacia en dicho propósito.

Como lo mencionaron los musicoterapeutas entrevistados por Cross (2014), este trabajo constata que el ritmo es un elemento imprescindible para dar estructura y clarificar el discurso tanto musical, como verbal, como escrito. La percepción del ritmo es un elemento clave para la discriminación auditiva y esa misma es la base de la conciencia fonológica. Los soportes visuales también fueron de gran ayuda a la hora de trabajar una actividad directamente ligada a la lectura. Asimismo, se verificó la importancia de diseñar unas actividades propias para cada grupo y así cumplir con las características y las necesidades de cada uno, aunque el objetivo general fuese el mismo en ambos casos.

\section{Conclusiones}

Según los resultados obtenidos de las evaluaciones, se confirma la hipótesis planteada al principio de este trabajo, que afirmaba que la musicoterapia contribuía la mejora de la precisión y de la velocidad lectora en niños y niñas escolarizados con dificultades de aprendizaje de edades comprendidas entre 6 y 9 años.

Más allá del desarrollo de las habilidades lectoras, las sesiones grupales pueden haber tenido un efecto positivo sobre la dinámica de grupo y la relación entre pares. Se trabajó la inhibición y la escucha a través del respeto de los turnos y del trabajo en grupo. Esas habilidades son importantes para este alumnado que suele tener poco espacio para expresarse debido a sus dificultades, y su fomento puede a su vez influir positivamente sobre la autoestima de estos niños y niñas.

Sin olvidar el trabajo llevado a cabo por el alumnado a lo largo de esos tres meses dentro y fuera del colegio por otros especialistas, se desea enfatizar en la importancia de inclusión de la musicoterapia dentro de un contexto multidisciplinario para poder lograr una mayor eficacia. 


\section{Referencias}

A.P.A. (2013). Diagnostic and statistical manual of mental disorders. Arlington: American Psychiatric Publishing.

Adams, M. J. (1990). Beginning to read: Thinking and learning about print. Cambridge, Mass: MIT Press.

Anvari, S. H., Trainor, L. J., Woodside, J., \& Levy, B. A. (2002). Relations among musical skills phonological processing, and early reading ability in preschool children. Journal of Experimental Child Psychology , 83, 111-130.

Bonacina, S., Krizman, J., White-Schwoch, T., Nicol, T., \& Kraus, N. (2019). How Rhythmic Skills Relate and Develop in School-Age Children. Global Pediatric Health, 6, 1-7.

Corriveau, K., \& Goswami, U. (2009). Rhythmic motor entrainment in children with speech and language impairments: taping to the beat. Cortex , 45, 119-130.

Cross, C. M. (2014). A Survey of Music Therapists' Use of Music to Facilitate Reading Development in Children. Master's Theses , 541.

Fuiza Azorey, M. J., \& Fernández Fernández, M. P. (2013). dificultades de aprendizajey trastornos del desarollo. Madrid: Ediciones Pirámide.

Goswami, U. (2013). Dyslexia - In tune but out of time. Psychologist , 26 (2), 106-109.

J. d., \& C. d. (2010). Guía de evaluación de destrezas lectoras. Educación Primaria. Sevilla: Agencia Andaluza de Evaluación Educativa.

Lorenzo, O., Herrera, L., Hernández-Candelas, M., \& Badea, M. (2014). Influence of music training on language development. A longitudinal study. Procedia - Social and Behavioral Sciences , 128, 527-530.

Martínez, M., Henao, G., \& Gómez, L. (2009). Comorbilidad del trastorno por déficit de atención e hiperactividad con los trastornos específicos del aprendizaje. Revista Colombiana de Psiquiatría, 38 (1), 178-194.

Moritz, C., Yampolsky, S., Papadelis, G., Thomson, J., \& Wolf, M. (2013). Links between early rhythm skills, musical training, and phonological awareness. Reading and Writing , 26 (5), 739-769.

Register, D. (2001). The Effects of an Early Intervention Music Curriculum on Prereading/Writing. Journal of Music Therapy, 38 (3), 239-248.

Sallat, S., \& Jentschke, S. (2015). Music Perception Influences Language Acquisition: Melodic and Rhythmic-Melodic Perception in Children with Specific Language Impairment. Behavioural Neurology, 2015, 1-10.

Sánchez, J., \& Hidalgo, M. D. (1990). Implicaciones de la codificación visual en el retraso específico en lectura. Psicothema , 2 (2), 35-48.

Santiuste, V., \& González-Pérez, J. (2005). Dificultades de aprendizaje y intervención psicopedagógica. Madrid: CCS.

Schwartz, S. (1984). Measuring reading competence. New York: Plenum Press.

Talamini, F., Grassi, M., Toffalini, E., Santoni, R., \& Carretti, B. (2018). Learning a second language: Can music aptitude or music training have a role? Learning and Individual Differences , 64, 1-7.

Tierney, A., \& Kraus, N. (2013). The ability to tap to a beat relates to cognitive, linguistic, and perceptual skills. Brain Lang , 124, 225-231. 
Thomson, J., \& Goswami, U. (2008). Rhythmic processing in children with developmental dislexia: auditory and motor rhythms link to reading and spelling. $J$ Physiol Paris , 102, 120-129.

Treiman, R. (1991). Phonological awarness and its rol in learning to read and spell. In D. Sawyer, \& B. Fox, Phonological awarness in reading: the evolution of current perspectives (pp. 159-189). New York: Springer-Verlag. 